\title{
The Influence of Airflow Characteristics and Accumulation Grid Velocity on the Formation of a Stone Wool Primary Layer
}

\author{
Marko Peternelj* - Benjamin Bizjan - Branko Širok \\ University of Ljubljana, Faculty of Mechanical Engineering, Slovenia
}

In this paper, the industrial process of stone wool primary layer formation has been investigated. The blow-away airflow, collecting chamber suction pressure, and peripheral velocity of collecting member effects on primary layer area density have been investigated. In total, 27 operating points have been measured by means of primary layer visualization. Computer-aided visualization has been carried out by two digital cameras. Each operating point was monitored at two locations: the first one at the beginning of fibre accumulation, forming the primary layer, and the secondary one at the end of the formation zone. The mass attenuation coefficient was calculated for each operating point and then used to calculate the primary layer area density. It was determined that primary layer bulk density distribution and primary layer texture are significantly influenced by accumulation grid peripheral velocity and blow-away airflow; however, suction pressure has a less pronounced effect on those characteristics. At the highest accumulation grid velocity and blow-away flow rate, the area density was $37 \%$ lower than at the lowest grid velocity and blow-away flow, with a corresponding increase in a standard deviation of $750 \%$. Multiple regression models suggest very good agreement with the measured data $\left(R^{2}=0.94\right.$ to 0.98$)$.

Keywords: mineral wool, fibre, primary layer formation, spinner, collecting chamber, pneumatic transport

Highlights

- Mineral wool primary layer formation has been investigated at a large-capacity industrial plant.

- $\quad$ Primary layer was observed at its formation stage and at the end, where it is completely formed.

- $\quad$ Primary layer bulk density distribution, its standard deviation, and texture are significantly affected by accumulation grid peripheral velocity and blow-away airflow.

- A very good correlation $\left(0.944<R^{2}<0.976\right)$ between measured data and multiple regression models for primary layer bulk density, its standard deviation and primary layer entropy was determined.

\section{O INTRODUCTION}

Mineral wool is a fibrous material commonly used for thermal and acoustic insulation and can be divided into subtypes, such as stone and glass wool. The production process of mineral wool is highly complex as it includes many physical phenomena at different lengths and time scales. To produce high quality mineral wool, a good understanding and control of several production phases is required.

Mineral wool quality is most significantly affected by the ratio of different raw materials, homogeneity of melt that is turned into fibres, fiberization process, pneumatic transport and deposition of fibres into the primary layer [1]. Additional processes that follow include overlaying the primary layer into the secondary layer, compressing and heating it in a curing oven to polymerize the binder added to the fibres.

Nowadays, the most commonly used method of forming fibres is the pouring of melt on solid fastspinning wheels with horizontal axes or pouring it into hollow perforated rotors with vertical axes. The first method is used mostly for stone wool and the second for glass wool. The fiberization process was a subject of several experimental and numerical studies for both solid wheel spinners (Širok et al. [1] and [2], Westerlund and Hoikka [3]) and perforated rotors (Panda [4], Qin et al. [5] and [6], Kraševec et al. [7] and [8]).

After fibres are formed, they are pneumatically transported by means of blow-away and suction airflows to the continuously moving accumulation grid where the primary layer of mineral wool is formed [1]. A good quality primary layer is defined by a low degree of spatial fluctuations in the thickness and density of the fibre deposit, and is very important for achieving the optimal insulation and mechanical properties of end products [1]. Thus far, measurement of aerodynamic characteristics and flow visualization were the main experimental approaches to investigating the primary layer formation process due to the high complexity of the multiphase nature of the flow.

The pneumatic transport of fibres in an axial airflow was modelled numerically by Lin et al. [9] and experimentally by Capone et al. [10] and Qi et al. [11]. In these studies, fibres were modelled as rigid bodies with relatively low aspect ratio (i.e. length to diameter ratio). Šrok et al. [1] showed that mineral wool fibres 
typically have aspect ratios in excess of 1000 , leading to complex flows with mutual intertwining and breakage of fibres, and also inhomogeneous primary layer deposited on the accumulation grid. Other experiments and monitoring of production process in the stone wool industrial environment were conducted by Blagojević et al. [12] and Bajcar et al. [13] who developed quantitative measures for the monitoring of the primary layer quality.

Numerous measures have been proposed to homogenize the blow-away flow velocity field by improved aerodynamic geometry of collecting chamber which reduce vortices and recirculation (Peternelj et al. [14]). Adding centrifugal rotors to the spinner wheels were proposed by Širok et al. [1] to reduce air velocity extremes at the accumulation grid, caused by the blow-away airflow.

This study was conducted as a further investigation of primary layer formation, already conducted by Bizjan et al. [15], where model spinning machine similar to the glass wool spinners was used. The primary layer formation in this study was investigated in an industrial stone wool plant and was also analysed by a visualization method, but at two stages simultaneously, first at the forming stage and second at the end of the formation zone. The primary layer quality was characterized by the bulk density distribution, its standard deviation and texture entropy. Multiple regression models were conducted to correlate the primary layer characteristics with the operating parameters.

Thus far, most experimental work on this subject was done in a laboratory environment in the form of simplified models, and alternative working medium was used. The bulk density and its distribution is one of the most crucial parameters for high quality product from stone wool. The weighing scales normally used for measurement of the mineral wool mass flow are not suitable for measuring the mass of the primary layer, as it is thin and low density, traveling at high velocities and would have to be dynamically isolated from the surroundings, which is very difficult or even impossible to achieve in industrial environment. Furthermore, many weighing scales would have to be placed along the width of primary layer to obtain good measurement of bulk density distribution. The main novelty in this paper is the optical measurements of primary bulk density, not only at the end of the formation, but also from the very start of formation and to its end. This provides a clearer understanding of primary layer formation.

This paper is organized as follows. The experimental setup section describes the mineral wool primary layer formation and the experimental setup used to study this process. In the theoretical background section the primary layer visualization method used in our experiments is explained whereas in the results and discussion section, the qualitative and quantitative results of the primary layer image visualization analysis are presented.

\section{EXPERIMENTAL SETUP}

Measurements were conducted at an industrial stone wool production plant. Fig. 1 presents the experimental setup used; the longitudinal cross section of drum

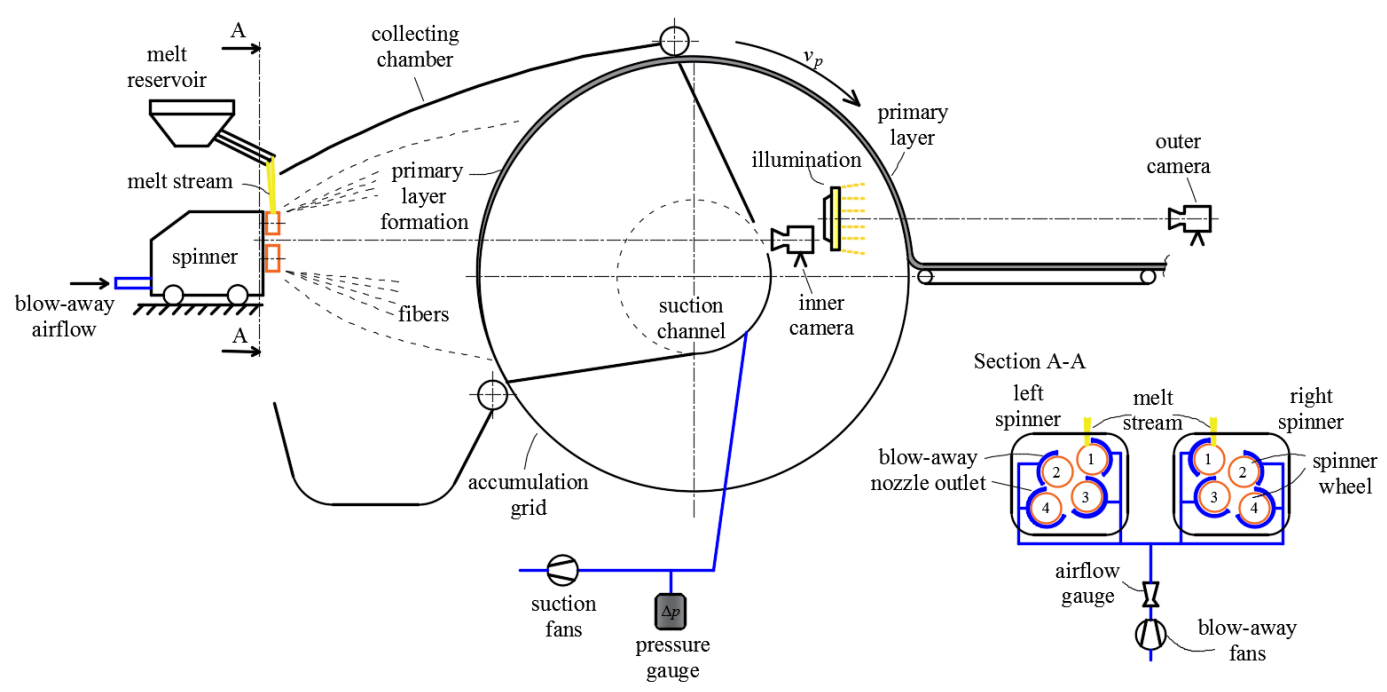

Fig. 1. Experimental setup of primary layer area density investigation 
type collecting chamber with fiberization machine and auxiliary systems is shown. Accumulation grid was a cylinder or drum type perforated sheet metal. Peripheral velocity of accumulation grid $v_{p}$ and consequently primary layer velocity was varied by a frequency inverter and was set to $88 \mathrm{~m} / \mathrm{min}$, $106 \mathrm{~m} / \mathrm{min}$ and $124 \mathrm{~m} / \mathrm{min}$, respectively.

The fiberization machine consists of two symmetrical spinners, each equipped with four rotating wheels on a horizontal axis.

Melt is poured on the first wheel of each spinner where, due to centrifugal force, it is partially ejected to other wheels and partially transformed into ligaments. Around each wheel, a blow-away airflow nozzle is mounted to transport ligaments away from the wheel and turn them into solid fibres. Blow-away airflow is generated by radial fans. The total blow-away airflow was set to the range of $13.69 \mathrm{~m}^{3} / \mathrm{s}$ to $17.35 \mathrm{~m}^{3} / \mathrm{s}$ (Table 1).

Secondary (suction) airflow was achieved via suction fans, which are all connected to one suction channel. Suction airflow helps collecting fibres on the accumulation grid, where they form the primary layer. A pressure gauge was installed in the suction channel to regulate suction fans speed so that the pressure inside the channel remained constant during particular operating points. Suction pressure $\Delta p$ was set to the range of $500 \mathrm{~Pa}$ to $800 \mathrm{~Pa}$.

Due to variations of the fibre mass flow rate, the secondary layer (overlapped primary layer) mass was measured using a weighing scale to calibrate light illumination absorption measuring method that was used later. The temperature of the ambient air during the experiments was between $0{ }^{\circ} \mathrm{C}$ and $10^{\circ} \mathrm{C}$. The temperature of the blow-away airflow was between $40{ }^{\circ} \mathrm{C}$ and $50{ }^{\circ} \mathrm{C}$, and the temperature in the suction channel varied in the range of $35^{\circ} \mathrm{C}$ to $50^{\circ} \mathrm{C}$. Due to the insignificant air temperature variability, the effects of temperature on the thermophysical properties of the air are not considered in this study. Temperature, dynamic viscosity, density and surface tension of melt were $1400{ }^{\circ} \mathrm{C}, 1.88 \mathrm{~Pa} \cdot \mathrm{s}$ [16], $2710 \mathrm{~kg} / \mathrm{m}^{3}$ [17] and [18] and $0.41 \mathrm{~N} / \mathrm{m}$ [19], respectively. The composition of input raw materials was not changed during experiments and the temperature of the melt from the furnace was also kept constant. The airflow velocity from blow-away nozzles was varied in the range of $100 \mathrm{~m} / \mathrm{s}$ to $150 \mathrm{~m} / \mathrm{s}$, gradually decreasing to the range of $4 \mathrm{~m} / \mathrm{s}$ to $10 \mathrm{~m} / \mathrm{s}$ at the accumulation grid. Considering the blow-away flow as an example of a flow around a cylinder, the Reynolds number can be estimated to the order of magnitude of $10^{7}$ in the entire pneumatic transport zone, meaning that the flow is turbulent everywhere.

Fig. 2 shows the final primary layer structure when it exits the collecting chamber. On the top and bottom of the image, nonilluminated (lower green rectangular) and illuminated (upper blue rectangular) areas are marked. Illumination was achieved by a light source from the inside of the accumulation grid facing to the acA800-200gc Basler outer camera. Fourteen LED lights in a side-by-side arrangement were used for this purpose. Each light required $100 \mathrm{~W}$ of electric power. $200 \mathrm{~mm}$ away from LED lights, a light diffusion plastic sheet was mounted to create a homogenous light source. Images were taken by the inner and the outer camera which both recorded at 10 frames per second.

Table 1 provides the 27 operating points obtained by all possible combinations of operating parameters.

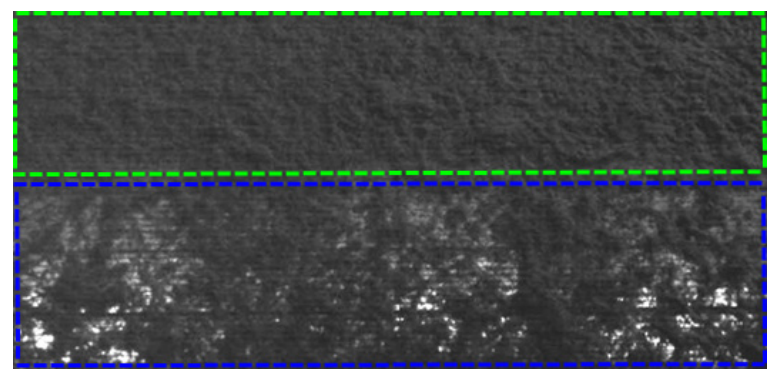

Fig. 2. Illuminated (blue (lower) frame) and nonilluminated (green (upper) frame) area of final primary layer

After steady state conditions of production had been achieved, measurement of the first operating point started. After 4 minutes, the values for the next operating point were set. To minimize the effect of transition between operating points due to the high inertia of fans, the accumulation grid peripheral velocity $v_{p}$ had been changed most frequently, followed by variations of suction pressure $\Delta p$ and at last the blow-away airflow $Q_{b}$ (Table 1). At each operating point, the primary layer was simultaneously recorded by the inner and the outer camera. All operating parameters were also measured and logged every 10 seconds, so the mean value of each parameter for each operating point could be calculated later. Analyses show that the transient effect was negligible after approximately $60 \mathrm{~s}$ in all operating points. The last 18 seconds of each operating point were also excluded in further analyses. As a result, imaging data for the remaining 162 seconds of each operating point were chosen for the analysis of primary layer properties. 
Table 1. Actual mean values of measured operating parameters for all operating points

\begin{tabular}{|c|c|c|c|c|c|}
\hline $\mathrm{OP}$ & $\begin{array}{c}\overline{v_{p}} \\
{[\mathrm{~m} / \mathrm{min}]}\end{array}$ & $\begin{array}{l}\overline{\Delta p} \\
{[\mathrm{~Pa}]}\end{array}$ & $\begin{array}{c}\overline{Q_{b}} \\
{\left[\mathrm{~m}^{3} / \mathrm{s}\right]}\end{array}$ & $\begin{array}{c}\overline{q_{p}} \\
{[\mathrm{~kg} / \mathrm{s}]}\end{array}$ & $\begin{array}{c}\overline{\mu_{A}} \\
{\left[\mathrm{~kg} / \mathrm{m}^{2}\right]}\end{array}$ \\
\hline 1 & 88 & 489 & 15.30 & 3.53 & 0.633 \\
\hline 2 & 106 & 486 & 15.30 & 3.39 & 0.505 \\
\hline 3 & 124 & 497 & 15.30 & 3.45 & 0.439 \\
\hline 4 & 124 & 646 & 15.30 & 3.38 & 0.430 \\
\hline 5 & 106 & 666 & 15.30 & 3.36 & 0.500 \\
\hline 6 & 88 & 642 & 15.30 & 3.40 & 0.611 \\
\hline 7 & 88 & 824 & 15.30 & 3.37 & 0.604 \\
\hline 8 & 106 & 785 & 15.30 & 3.55 & 0.529 \\
\hline 9 & 124 & 790 & 15.30 & 3.55 & 0.452 \\
\hline 10 & 124 & 788 & 17.35 & 3.29 & 0.418 \\
\hline 11 & 106 & 798 & 17.35 & 3.29 & 0.490 \\
\hline 12 & 88 & 811 & 17.35 & 3.41 & 0.612 \\
\hline 13 & 88 & 654 & 17.35 & 3.41 & 0.611 \\
\hline 14 & 106 & 633 & 17.35 & 3.26 & 0.486 \\
\hline 15 & 124 & 641 & 17.35 & 3.39 & 0.432 \\
\hline 16 & 124 & 501 & 17.35 & 3.31 & 0.421 \\
\hline 17 & 106 & 509 & 17.35 & 3.29 & 0.490 \\
\hline 18 & 88 & 515 & 17.35 & 3.41 & 0.613 \\
\hline 19 & 88 & 530 & 13.69 & 3.61 & 0.647 \\
\hline 20 & 106 & 482 & 13.69 & 3.53 & 0.526 \\
\hline 21 & 124 & 490 & 13.69 & 3.59 & 0.458 \\
\hline 22 & 124 & 648 & 13.69 & 3.62 & 0.461 \\
\hline 23 & 106 & 654 & 13.69 & 3.78 & 0.562 \\
\hline 24 & 88 & 670 & 13.69 & 3.72 & 0.667 \\
\hline 25 & 88 & 816 & 13.69 & 3.81 & 0.683 \\
\hline 26 & 106 & 797 & 13.69 & 3.73 & 0.555 \\
\hline 27 & 124 & 792 & 13.69 & 3.64 & 0.463 \\
\hline
\end{tabular}

In Fig. 3, three sample images of the illuminated primary layer are shown for different operating settings. A significant effect of the operating parameters on the primary layer structure can be seen. In Fig. 3a, the lowest accumulation grid velocity and blow-away airflow is used at the maximum suction pressure, resulting in the most uniform bulk density distribution of primary layer. From Fig. $3 a$ to c, the accumulation grid velocity and the blow-away airflow are increased, while the suction pressure is decreased, causing larger inhomogeneities in this order.

Fig. 4 shows three images of the primary layer formation on the accumulation grid for the same operating points as shown in Fig. 3. On each image two darker zones can be seen which are caused by higher fibre density in front of each spinner. Accumulation grid movement direction is shown and according to image gray level intensity, the primary layer density increases from the bottom to the top.

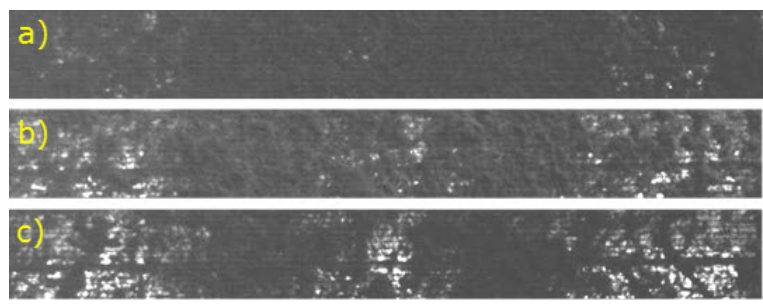

Fig. 3. Illuminated primary layer image taken by outer camera at operating points; a) $v_{p 1}-\Delta p_{3}-\mathrm{Q}_{b 1}$; b) $v_{p 2}-\Delta p_{2}-\mathrm{Q}_{b 2}$; c) $v_{p 3}-\Delta p_{1}-\mathrm{Q}_{b 3}$

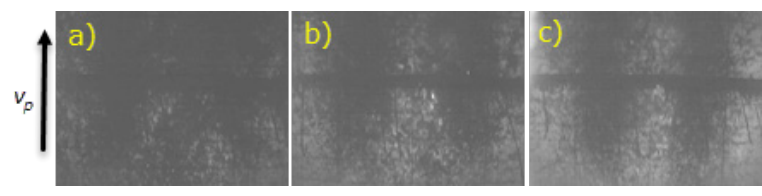

Fig. 4. Primary layer image taken by inner camera at operating points; a) $v_{p 1}-\Delta p_{3}-\mathrm{Q}_{b 1}$; b) $v_{p 2}-\Delta p_{2}-\mathrm{Q}_{b 2}$;

$$
\text { c) } v_{p 3}-\Delta p_{1}-\mathrm{Q}_{b 3}
$$

\section{THEORETICAL BACKGROUND}

The illuminated area in the image was horizontally split into 21 subsections (Fig. 5) and for each subsection, the average gray level $I_{i l l, i j}$ was calculated, where index $i$ states image in a sequence and $j$ subsection from the left to right side of the image.

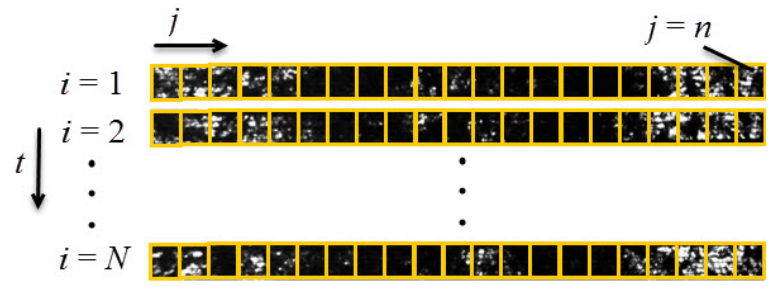

Fig. 5. Illuminated area subsections

To include background light variations, an additional area of the image was taken just below the illuminated area, where an average referenced gray level $I_{r e f, i j}$ was calculated. As the grayscale images taken are 8-bit there are 256 gray levels and corrected gray level $I_{i j}$ can be defined as:

$$
I_{i j}=1-\frac{\left(I_{i l l, i j}-I_{r e f, i j}\right)}{255-I_{\text {ref }, i j}},
$$

and the normalized gray level as:

$$
I_{n, i j}=\frac{I_{i j}}{\frac{1}{n} \sum_{j=1}^{n} I_{i j}} .
$$


A linear correlation between the normalized gray level $I_{n, i j}$ and the primary layer area density $\mu_{A, i j}$ was used:

$$
\mu_{A, i j}=\overline{\mu_{A}} \cdot I_{n, i j},
$$

where $\overline{\mu_{A}}$ is the average primary layer area density:

$$
\overline{\mu_{A}}=\frac{\overline{q_{p}}}{v_{p} B},
$$

and $\overline{q_{p}}, v_{p}$ and $B$ are the measured average mass flow rate, velocity and width of primary layer, respectively. The primary layer area density in normalized form is then:

$$
\mu_{n, i j}=\frac{\mu_{A, i j}}{\overline{\mu_{A}}}=I_{n, i j} .
$$

To analyse the primary layer area density $\mu_{A}$ in formation stage, the Beer-Lambert law was used:

$$
\mu_{A, i, j}=\frac{\ln \left(\bar{I}_{i, j} / I_{0}\right)}{\alpha_{m}},
$$

where $\bar{I}_{i, j}$ is the temporally averaged gray level of the observed section, $I_{0}$ is the grey level of image background (accumulation grid without fibres) and $\alpha=5.8 \mathrm{~m}^{2} / \mathrm{kg}$ is the mean mass attenuation coefficient, calculated for the illuminated primary layer observed with the inner camera.

\section{RESULTS AND DISCUSSION}

\subsection{Primary Layer Mass Distribution Analysis}

Fig. 3 displays 9 diagrams showing the relationship between the normalized primary layer area density $\mu_{n}$ and the normalized coordinate $x / B$ at different operating points. There are three curves on each diagram representing the normalized primary layer area density $\mu_{n}$ at different accumulation grid velocity $v_{p}$, specific suction pressures $\Delta p$ and blowaway airflows $Q_{b}$. Diagrams are arranged so that the intensity of suction pressure $\Delta p$ increases from left to right, and the intensity of blow-away airflow $Q_{b}$ increases from top to bottom.

By comparing different operating parameters, it can be concluded that in all cases the normalized primary layer area density $\mu_{n}$ is significantly larger at intervals $0.2<x / B<0.4$ and $0.6<x / B<0.8$ where a convex shaped curve is formed with the maximum value at the middle of each interval. This increase is caused by the position of the spinner at this coordinate. Due to the higher concentration of fibres coaxially from the spinner, the primary layer area density $\mu_{A}$ is increased at those areas. At remaining intervals, a concave shaped curve is formed with the minimum value also approximately in the middle of each interval.
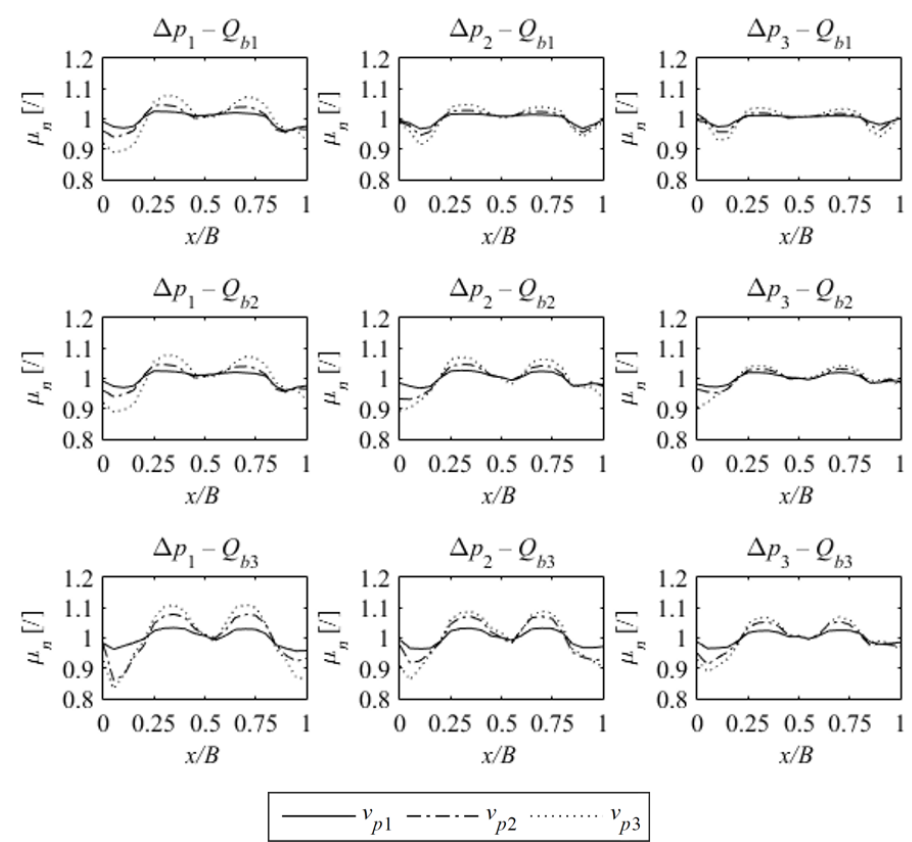

Fig. 6. Relationship between the normalized primary layer area density $\mu_{n}$ and the normalized coordinate $x / B$ (0 - left, 1 - right) at different operating points (see Table 2) 
For all diagrams, it can be concluded that the increase of accumulation grid velocity $v_{p}$, increases differences between maximum and minimum normalized primary layer area densities, which can be explained by the fact that a faster accumulation grid causes thinner primary layer inside the collecting chamber and the area on the accumulation grid directly in front of the spinner is less filled by fibres, and the airflow with fibres is not redirected on accumulation grid sides.

A similar effect can be seen by increasing the blow-away airflow $Q_{b}$, especially in the first column of diagrams, where the blow-away airflow $Q_{b}$ inside the collecting chamber is increased from top to bottom. In this case, the increase between extreme normalized primary layer area densities can be explained by the fact that the coaxial velocity of the blow-away airflow is significantly higher at the spinner centre and it quickly decreases when moving transversally to off-centre positions. Consequently, at high blowaway airflows, the main fibre flow is concentrated closer to the spinner centre projected horizontally to the accumulation grid, and at its high $v_{p}$, this effect is additionally magnified. This can be seen in the bottom left diagram $\left(\Delta p_{1}=500 \mathrm{~Pa}, Q_{b 3}=17.35 \mathrm{~m}^{3} / \mathrm{s}\right)$, where at the accumulation grid velocity $v_{p 3}=124 \mathrm{~m} / \mathrm{min}$, difference between $\mu_{n, \max }$ and $\mu_{n \text {,min }}$ is about $25 \%$.

An increase of suction pressure $\Delta p$ decreases the primary layer area density fluctuations along coordinate $x$ as can be seen comparing diagrams from left to right. This is due to the fact that the airflow field caused by suction fans varies less in the transversal direction on the accumulation grid compared to coaxial blow-away airflow out of the spinner nozzles. Increasing the suction pressure $\Delta p$ homogenizes the flow velocity field in the collecting chamber, causing fibres to spread more evenly along the width of the accumulation grid (coordinate $x$ ).

For a more in-depth comparison of the results presented in diagrams in Fig. $6, \sigma_{\mu n}$ is defined as a standard deviation of the normalized primary layer area density $\mu_{n}$, where $\mu_{n}$ is a function of the normalized coordinate $x / B$. Results are presented in Fig. 7 where a relation between standard deviation $\sigma_{\mu n}$ and operating parameters settings are shown. It can be concluded that an increase of the accumulation grid velocity and of the blow-away airflow leads to a larger inhomogeneity of the primary layer area density along the width of the accumulation grid. In contrast, an increase of the suction pressure improves the homogeneity of the primary layer area density distribution.

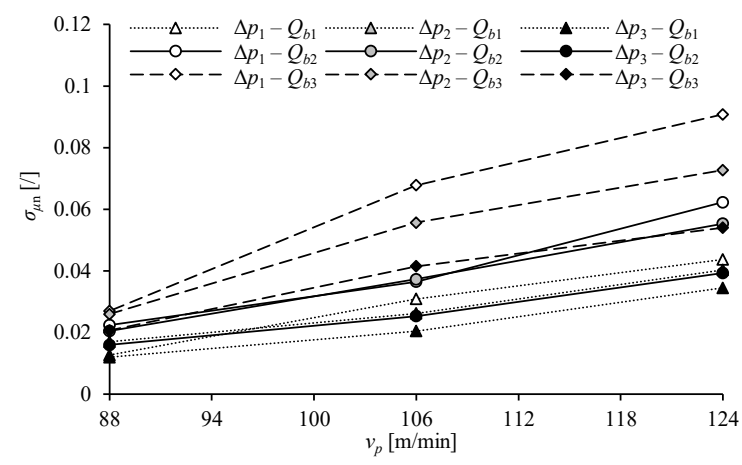

Fig. 7. Standard deviation $\sigma_{\mu n}$

\subsection{Primary Layer Formation inside Collecting Chamber}

In Fig. 8 there are 9 diagrams of area density $\mu_{A}$ distribution at the forming stage in the collection chamber. The diagrams are arranged so that the parameter $v_{p}$ increases from left to right and $Q_{b}$ from top to bottom. As it turns out, these two parameters have the most significant influence on area density distribution so parameter $\Delta p$ is constant for all diagrams in Fig. 8. By a qualitative comparison of diagrams, one can conclude that by increasing accumulation grid velocity, area density $\mu_{A}$ decreases and the bottom area with almost no fibre is increased. This area of fibre-free accumulation grid also represents energy losses due to suction flow through this area. At the topright diagram $\left(v_{p 3}-\Delta p_{3}-Q_{b 1}\right)$ around half of the observed area is practically without fibres. By increasing the blow-away airflow, the area density $\mu_{A}$ increases at spinner centre vertical planes $(x / B \approx 0.3$ and $x / B \approx 0.7)$. This effect is clearly visible in the bottomleft diagram, representing lowest $v_{p}$ and highest $Q_{b}$ operating points.

\subsection{Texture Analysis of the Primary Layer}

The image texture was analysed using the image entropy function, which is a statistical measure for randomness [20]. At high values of entropy, more random texture of primary layer is expected compared to low entropy values where the primary layer texture is more uniform and homogenous. Image grayscale entropy is defined as:

$$
E=-\sum_{k=0}^{M} p_{k} \log _{2}\left(p_{k}\right)
$$

where $M$ is the number of gray levels and $p_{k}$ is the probability associated with gray level $k$. For the texture analysis, a nonilluminated image area has been 

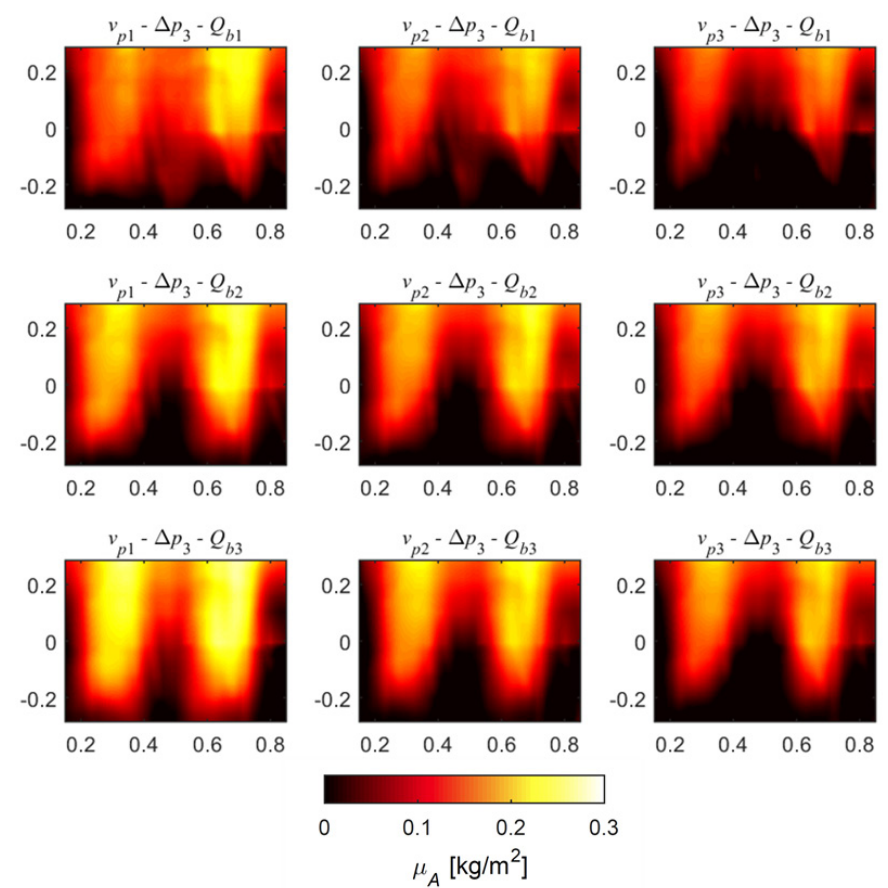

Fig. 8. Area density $\mu_{A}$ at primary layer forming stage for different operating points (vertical and horizontal axes on each diagram represents normalized coordinate $y / B$ and $x / B$, respectively)

chosen to calculate the entropy $E$. Furthermore, the mean value of entropy $E$ (Eq. (7)) for each operating point was calculated and normalized to its maximum value (Eq. (8)).

$$
E_{n, O P}=\frac{\overline{E_{O P}}}{E_{\max , O P}},
$$

The dimensional entropy $E$ (Eq. (7)) was affected not only by the layer texture itself, but also by the mean brightness level, which varied slightly between operating points. The entropy $E$ was normalized to its maximum value to eliminate brightness level variations, so direct comparison between operating points was possible. Results are presented in diagram in Fig. 9, where we can see that by decreasing suction pressure and even more significantly by increasing accumulation grid velocity, normalized entropy $E_{n}$ decreases, meaning that the primary layer texture is less random.

At the lowest accumulation grid velocities, minimum entropy is obtained at medium blow-away airflow in all three cases of suction pressure, however, in all other cases normalized entropy decreases by increasing the blow-away airflow. According to the image grayscale, normalized entropy $E_{n}$ maximum and minimum randomness of primary layer texture is obtained at operating parameters $v_{p 1}-\Delta p_{3}-\mathrm{Q}_{b 1}$ $\left(E_{n}=0.34\right)$ and $v_{p 3}-\Delta p_{1}-\mathrm{Q}_{b 3}\left(E_{n}=0.13\right)$, respectively.

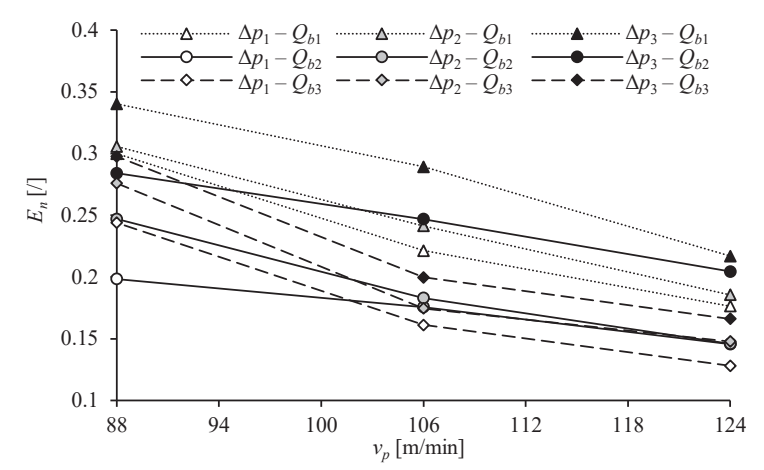

Fig. 9. Normalized entropy

Higher normalized entropy at lower accumulation grid velocities can be explained by the fact that at low accumulation grid velocity, the area in front of the spinner has a high fibre concentration and consequently, a lower permeability. Coaxial blowaway airflow starts to tear apart the already formed primary layer and forming bigger clusters of fibres. As the accumulation grid velocity increases, the permeability of the accumulation grid increases, causing a finer and homogenous texture, indicated by low normalized entropy as a parameter. 
To investigate texture dynamics, the DC component of entropy was eliminated from the signal so only the AC component was retained. A low-pass filter was used to reduce the high frequency noise in the signal. Power spectra are shown in Fig. 10, where peak amplitude is visible at low frequencies (bellow $0.2 \mathrm{~Hz}$ ) and can be correlated with accumulation grid angular frequency and its multiples.
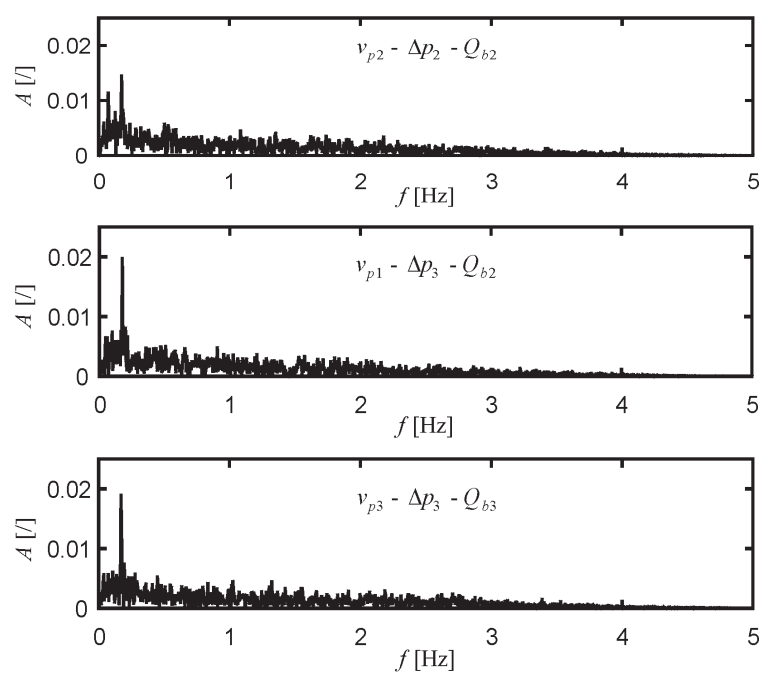

Fig. 10. Power spectrum of AC component of entropy $E_{n}$ for three operating points

\subsection{Multiple Regression Models}

A strong correlation between operating parameters $\left(\Delta p, Q_{b}, v_{p}\right.$ and $\left.q_{p}\right)$ and primary layer characteristic properties $\left(\overline{\mu_{A}}, \sigma_{\mu n}\right.$ and $\left.E_{n}\right)$ is expected and the power law multiple regression models (Eq. (9)) were used to evaluate the effect of each parameter.

$$
\Pi_{i}=a_{i} \cdot v_{p}^{b_{i}} \cdot Q_{b}^{c_{i}} \cdot \Delta p^{d_{i}} \cdot q_{p}^{e_{i}} .
$$

The exponents $a_{i}-e_{i}$ were determined by fitting the power law models in Eq. (9) to the measured data. The results are presented in Table 2, Figs. 11 to 13 where it is evident that most of the measurements are in good agreement with the proposed model.

Table 2. Regression model constant values

\begin{tabular}{cccc}
\hline & $\overline{\mu_{A}}$ & $\sigma_{\mu n}$ & $E_{n}$ \\
\hline $\mathrm{a}$ & 0.0524 & $2.361 \cdot 10^{-8}$ & 0.868 \\
\hline $\mathrm{b}$ & -0.740 & 2.919 & -1.337 \\
\hline $\mathrm{c}$ & 1.012 & 2.287 & -0.261 \\
\hline $\mathrm{d}$ & 0.130 & -0.686 & 0.471 \\
\hline $\mathrm{e}$ & 0.670 & -1.014 & 1.987 \\
\hline \hline$R^{2}$ & 0.944 & 0.976 & 0.958 \\
\hline
\end{tabular}

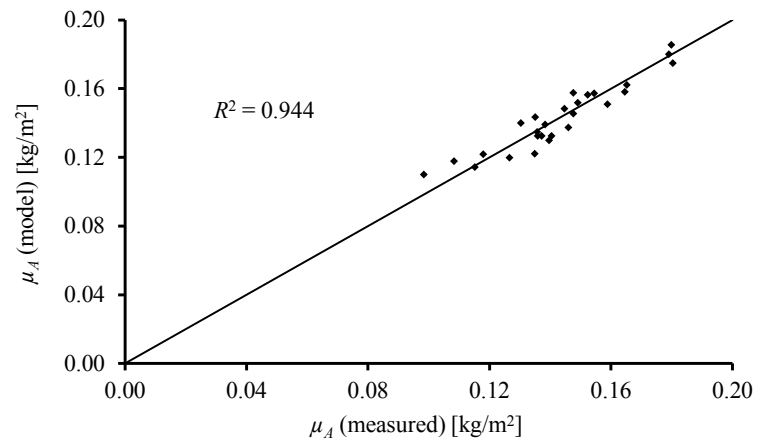

Fig. 11. Correlation between measured and modelled values of mean $\mu_{A}$ at observed forming stage

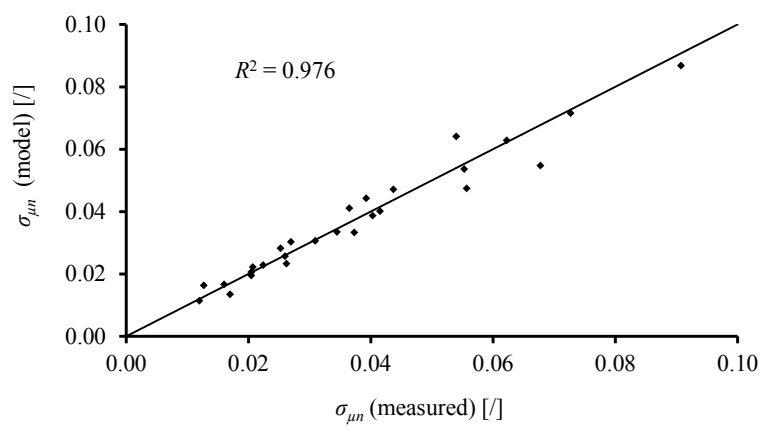

Fig. 12. Correlation between measured and modelled values of $\sigma_{\mu n}$ of completely formed primary layer

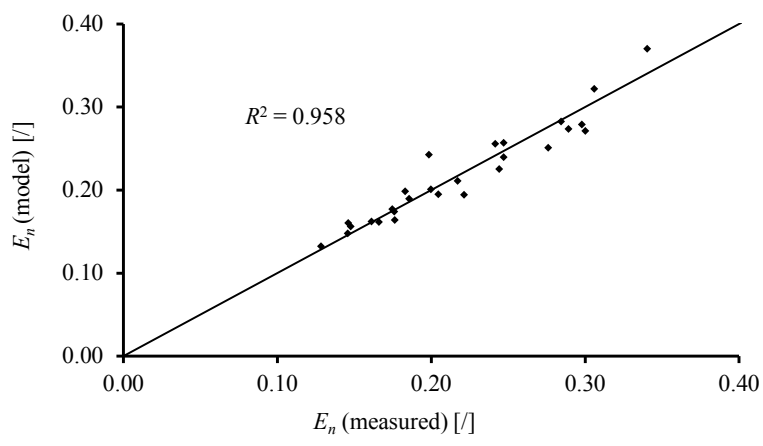

Fig. 13. Correlation between measured and modelled values of $E_{n}$ of completely formed primary layer

\section{CONCLUSIONS}

The process of continuous mineral wool primary layer formation was studied experimentally on a large capacity industrial plant comprising two spinning machines placed side-by-side. The process was investigated at two stages, first at the early stage of the primary layer formation inside the collecting chamber and at the completely formed primary layer at the exit from the collecting chamber. The primary layer 
area density was quantified with the light absorption method.

The area density, which should be as low and as uniform as possible, was found to significantly depend on all observed operating parameters $\left(v_{p}, \Delta p\right.$ and $\left.Q_{b}\right)$. A local increase in area density is caused by the higher intensity of the blow-away airflow, which causes an intense fibre concentration coaxially from the spinner. Lower and more uniform area density is achieved at higher accumulation grid velocities $v_{p}$. However, high $v_{p}$ cause smaller suction flow yield, because a large portion of the accumulation grid in the collecting chamber remains free of fibres, resulting in the suction flow passing this area. Another important measure for the primary layer quality is the homogeneity of its structure, which was quantified by the normalized standard deviation $\left(\sigma_{\mu n}\right)$ of the layer area density and by the layer entropy $\left(E_{n}\right)$. A low value of $\sigma_{\mu n}$ and $E_{n}$ is desired as it implies a good uniformity of the primary layer thickness and structure. It was determined that $\sigma_{\mu n}$ increases at higher $v_{p}$ and $Q_{b}$ and decreases at higher $\Delta p$. However, the opposite trend was found for the layer entropy $E_{n}$, which decreases at higher $v_{p}$ and $Q_{b}$ and increases at higher $\Delta p$. For all estimators, multi-regression models were carried out, where a very good agreement between measured data and model was obtained: $R_{\mu_{A}}^{2}=0.944, R_{\sigma_{\mu n}}^{2}=0.976$ and $R_{E_{n}}^{2}=0.958$.

Further experimental and numerical observations of the primary layer formation process will improve proposed models and implementing them into process control automatization in order to produce higher quality insulation materials with less energy and raw material consumed.

\section{NOMENCLATURE}

$B$ accumulation grid width, [m]

$E$ image grayscale entropy, [/]

$E_{n}$ normalized image grayscale entropy, [/]

$I_{0}$ gray level of image background, [/]

$I_{i l l}$ gray level of image illuminated, area, [/]

$I_{n}$ normalized gray level, [/]

$I_{\text {ref }}$ reference gray level, [/]

$M$ number of gray levels, [/]

$p_{k} \quad$ probability associated with gray level $k,[/]$

$Q_{b}$ total blow-away airflow, $\left[\mathrm{m}^{3 / \mathrm{s}}\right]$

$Q_{\text {in }}$ inner blow-away airflow, $\left[\mathrm{m}^{3} / \mathrm{s}\right]$

$Q_{\text {out }}$ outer blow-away airflow, $\left[\mathrm{m}^{3} / \mathrm{s}\right]$

$q_{p}$ primary layer mass flow rate, $[\mathrm{kg} / \mathrm{s}]$

$v_{p}$ accumulation grid peripheral velocity, $[\mathrm{m} / \mathrm{min}]$

$x$ distance from left image edge, $[\mathrm{m}]$

$y$ distance from bottom image edge, $[\mathrm{m}]$ $\alpha \quad$ mass attenuation coefficient, $\left[\mathrm{m}^{2} / \mathrm{kg}\right]$

$\Delta p$ suction pressure, $[\mathrm{Pa}]$

$\mu_{A}$ primary layer area density, $\left[\mathrm{kg} / \mathrm{m}^{2}\right]$

$\mu_{n} \quad$ normalized primary layer area density, [/]

$\sigma_{\mu n}$ standard deviation of $\mu_{n},[/]$

\section{REFERENCES}

[1] Širok, B., Blagojević, B., Bullen, P. (2008). Mineral Wool: Production and Properties. Woodhead Publishing, Cambridge, DOI:10.1533/9781845694456.

[2] Širok, B., Blagojević, B., Novak, M. (2002). Influence of blow away velocity field on the primary layer fibre structure in the mineral wool production process. Glass Technology, vol. 43, no. 5, p. 188-194.

[3] Westerlund, T., Hoikka, T. (1989). On the modeling of mineral fiber formation. Computers \& Chemical Engineering, vol. 13, no. 10, p. 1153-1163, Dol:10.1016/0098-1354(89)87018-8.

[4] Panda, S. (2006). The Dynamics of Viscous Fibers. PhD Thesis, TU Kaiserslautern, Kaiserslautern.

[5] Qin, Y., Lv, X., Bai, C., Chen, P., Qiu, G. (2013). Dry granulation of molten slag using a rotating multi-nozzle cup atomizer and characterization of slag particles. Steel Research International, vol. 84, no. 9, p. 852-862, D0l:10.1002/srin.201200325.

[6] Qin, Y., Lv, X., Bai, C., Chen, P., Qiu, G., Jie, Z. (2014). Mechanism of dry molten slag granulation using a rotating multi-nozzle cup atomizer. Steel Research International, vol. 85, no. 1, p. 44-52, Dol:10.1002/srin.201300007.

[7] Kraševec, B., Širok, B., Hočevar, M., Bizjan, B. (2014). Multiple Regression Model of Glass Wool Fibre Thickness on a Spinning Machine. Glass Technology, vol. 55, no. 4, p. 119-125.

[8] Kraševec, B., Širok, B., Bizjan, B., Hočevar, M. (2015). Fibre density distribution in a layer of glass wool. Glass Technology, vol. 56, no. 5, p. 145-152.

[9] Lin, J.Z., Liang, X.Y., Zhang, S.L. (2012). Numerical simulation of fiber orientation distribution in round turbulent jet of fiber suspension. Chemical Engineering Research and Design, vol. 90, no. 6, p. 766-775, D0l:10.1016/j.cherd.2011.09.016.

[10] Capone, A., Romano, G.P., Soldati, A. (2015). Experimental investigation on interactions among fluid and rod-like particles in a turbulent pipe jet by means of particle image velocimetry. Experiments in Fluids, vol. 56, paper no. 1, D0l:10.1007/ s00348-014-1876-4.

[11] Qi, G., Nathan, J.G., Lau, C.W.T. (2015). Velocity and orientation distributions of fibrous particles in the near-field of a turbulent jet. Powder Technology, vol. 276, p. 10-17, D0l:10.1016/j. powtec.2015.02.003.

[12] Blagojević, B., Širok, B., Hočevar, M. (2003). Monitoring and control of quality of the primary layer of mineral wool on a disc spinning machine. Instrumentation Science \& Technology, vol. 31, no. 1, p. 67-80, D0l:10.1081/Cl-120018408.

[13] Bajcar, T., Blagojević, B., Širok, B., Dular, M. (2007). Influence of flow properties on a structure of a mineral wool primary layer. Experimental Thermal and Fluid Science, vol. 32, no. 2, p. 440-449, DOI:10.1016/j.expthermflusci.2007.05.007.

[14] Peternelj, M., Mihalič, P., Širok, B. (2015). Collecting chamber and fiber formation method. PCT/SI2014/000016, World Intellectual Property Organization, Geneva. 
[15] Bizjan, B., Peternelj, M., Širok, B. (2016). Mineral Wool Primary Layer Formation in Collecting Chamber. Strojniški vestnik Journal of Mechanical Engineering, vol. 62, no. 3, p. 179-186, DOI:10.5545/sv-jme.2015.2995.

[16] Urbain, G. (1987). Viscosity estimation of slags. Steel Research International, vol. 58, no. 3, p. 111-116, Dol:10.1002/ srin.198701513.

[17] Bottinga, Y., Weill, D., Richet, P. (1982). Density calculations for silicate liquids. I. Revised method for aluminosilicate compositions. Geochimica et Cosmochimica Acta, vol. 46, no. 6, p. 909-919, D0I:10.1016/0016-7037(82)90047-3.
[18] Allibert, M., Gaye, H., Geiseler, J., Janke, D., Keene, B.J., Kirner, D., Kowalski, M., Lehmann, J., Mills, K.C., Neuschütz, D., Parra, R., Saint-Jours, C., Spencer, P.J., Susa, M., Tmar, M., Woermann, E. (2012). Slag atlas, 2nd ed., Verlag Stahleisen, Düsseldorf.

[19] Kucuk, A., Clare, A.G., Jones, L. (1999). An estimation of the surface tension of silicate glass melts at $1400{ }^{\circ} \mathrm{C}$ using statistical analysis. Glass Technology, vol. 40, no. 5, p. 149153.

[20] Gonzalez, R.C., Woods, R.E., Eddins, S.L. (2003). Digital Image Processing Using MATLAB, Prentice Hall, New Jersey, 2003, chapter 11, p. 535-598. 